\title{
BAYER PATTERN BASED DIGITAL ZOOMING APPROACH
}

\author{
R. Lukac and K.N. Plataniotis \\ Bell Canada Multimedia Laboratory, The Edward S. Rogers Sr. Department of ECE, \\ University of Toronto, 10 King's College Road, Toronto, Canada \\ lukacr@ieee.org,kostas@dsp.utoronto.ca
}

\begin{abstract}
In this paper, a new zooming algorithm suitable for singlesensor digital cameras is introduced. The proposed method is capable of zooming and enlarging Bayer data acquired by single-sensor cameras. The approach allows for operations on noise-free data at the hardware level. Complexity and cost implementation are thus greatly reduced. The proposed method utilizes a color difference model and an adaptive edge sensing mechanism capable of tracking the underlying structural content of the Bayer data. Simulation studies indicate that the new method yields excellent performance, in terms of both subjective and objective image quality measure.
\end{abstract}

\section{INTRODUCTION}

The commercial proliferation of single-sensor digital cameras and their use in novel applications such as wireless phones, sensor networks, and pocket devices has increased the demand for new algorithmic and technical developments in the area of color array (CFA) filtering, interpolation and zooming. The Bayer pattern (Fig. 1), the most widely used CFA, provides an array or mosaic of Red (R), Green (G) and Blue (B) colors in which only one color element is available at each spatial location. By allocating twice more spatial samples to the Green plane, the Bayer pattern improves the perceived sharpness of the digital image since it is wellknown that the human visual system (HVS) is more sensitive to luminance which is composed primarily of green light. The missing color components are recovered from the adjacent Bayer data using the so-called CFA interpolation or demosaicing process [7].

Conventional single-sensor digital cameras have enhanced optical/digital zooming capabilities providing the enduser with a variety of options, including recording modes, pre-determined lighting scenarios, storing formats and basic color image processing functions. Increasing the spatial resolution of the image is one of the most commonly performed re-processing operations. Since the cost of digital cameras rapidly increases with optical zooming and Megapixel capturing capabilities, to keep cost at a reasonable

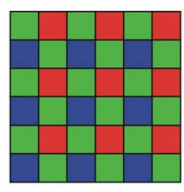

Fig. 1. Bayer CFA pattern.

level, camera manufacturers produce cameras capable of performing digital zooming.

It has to be said that the spatial resolution of the acquired, displayed and transmitted color images is conventionally performed in the RGB domain [3]. However, image zooming approaches [3],[6] as well as other image processing steps such as filtering [4] and compression [5], are more computationally efficient when applying to the CFA data. It is not difficult to see that by performing zooming, or processing in general, in the scalar values of the Bayer pattern rather than in the RGB vector space additional savings in terms of computational cost and power can occur [5]. As the result of CFA zooming, the enlarged CFA mosaic serves as the input to the demosaicing process producing a full color output image of increased spatial resolution.

\section{PROPOSED METHOD}

Let us consider a $K_{1} / k \times K_{2} / k$ Bayer image $z^{\prime}$ depicted in Fig.1, with the spatial coordinates $p, q$ denoting the image row and column. The Bayer image $z^{\prime}$ represents the input data acquired using a single-sensor digital camera. Zooming the Bayer data $z^{\prime}$ with a factor of $k$, this process results in a $K_{1} \times K_{2}$ zoomed Bayer image $z$. The zooming factor $k \in Z$ can be an arbitrary positive integer. The proposed method works for every $k>1$, however the value $k=2$ is selected here to facilitate the discussion.

Conventional zooming by a factor of 2 , proceeds first by enlarging the original image size and then incorporating new rows and columns (e.g. of zeros) into the original image. Operating on the Bayer data in this way destroys the structure of the Bayer pattern, since original values of $z^{\prime}$ oc- 


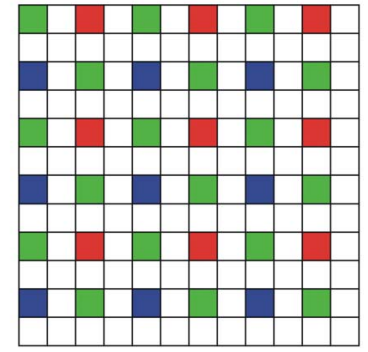

(a)

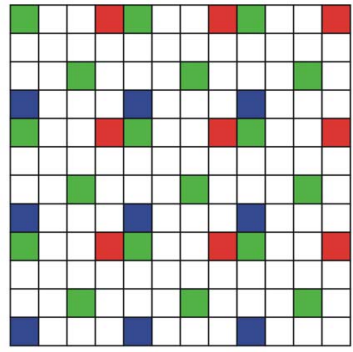

(b)
Fig. 2. The first Bayer image zooming step performed by: (a) conventional zooming approach, (b) the proposed method.

cupy spatial positions corresponding to other color channels of the enlarged image $z$. For example, as it can be seen in Fig. $2 a$ the conventional approach of Fig. $2 a$ stores R and B components in positions reserved for $\mathrm{G}$ samples.

To zoom the Bayer data and preserve the Bayer pattern structure, the original CFA data should be assigned unique positions, which correspond to the CFA color structure of the enlarged image as shown in Fig.2b. Using the proposed approach, the original $z^{\prime}$ values are filled into the zoomed image $z$ as follows:

$$
\left.\begin{array}{l}
z_{(2 p-1,2 q)} \\
z_{(2 p, 2 q-1)} \\
z_{(2 p-1,2 q-1)}
\end{array}\right\}=z_{(p, q)}^{\prime}, \begin{aligned}
& \text { for } p \text { odd and } q \text { even } \\
& \text { otherwise }
\end{aligned}
$$

where $p$ and $q$ denotes the coordinates in the original (small) Bayer image $z^{\prime}$.

Upon completion of this step, the enlarged Bayer image $z$, shown in Fig.2b, contains all the original CFA data of $z^{\prime}$. The zooming process continues by interpolating the missing CFA data corresponding to the empty positions of $z$ shown in Fig.2b. Considering an arbitrary spatial location $(p, q)$ in the image $z$ we can observe that the missing $\mathrm{G}$ components are surrounded by four original $\mathrm{G}$ components which form a diamond-shape mask on the image lattice. On the other hand, the four original R (or B) components create the square-shape mask on the image lattice. Since the missing component and its four surrounding corresponding original color components form a unique spatial arrangement, it is reasonable to interpolate the missing center using its corresponding color neighbors.

Let us consider the enlarged Bayer image $z$ of Fig.2b with the spatial coordinates $(p, q)$. The missing color component $z_{(p, q)}$ is positioned in the center of the four surrounding color components $W_{(p, q)}=\left\{z_{1}, z_{2}, z_{3}, z_{4}\right\}$. In the case of the $\mathrm{G}$ components, the quantities $z_{1}, z_{2}, z_{3}$ and $z_{4}$ are equivalent to corners $z_{(p-2, q)}, z_{(p, q-2)}, z_{(p, q+2)}$ and $z_{(p+2, q)}$, respectively, of a diamond-shape shown in Fig.3a. In the case of the $\mathrm{R}$ (or B) components, $z_{1}, z_{2}, z_{3}$ and $z_{4}$ are

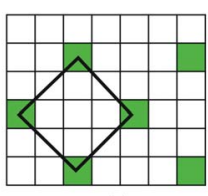

(a)

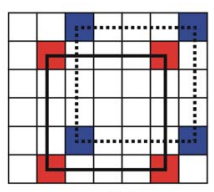

(b)
Fig. 3. Geometrical shapes determined by the Bayer pattern structure: (a) a diamond-shape, (b) a square-shape.

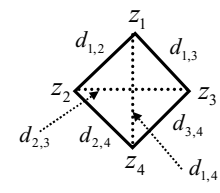

(a)

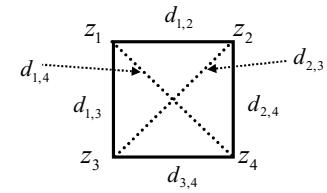

(b)
Fig. 4. Component and distance arrangement: (a) a diamond-shape, (b) a square-shape.

equivalent to components $z_{(p-2, q-2)}, z_{(p-2, q+2)}, z_{(p+2, q-2)}$ and $z_{(p+2, q+2)}$, respectively, which create the square-shape masks depicted in Fig.3b.

In real images, color components $z_{1}, z_{2}, z_{3}, z_{4}$ vary in values, therefore it is necessary to differentiate their influence interpolating the missing component $z_{(p, q)}$. Therefore, the difference between any $z_{i} \in W_{(p, q)}$ and $z_{j} \in W_{(p, q)}$ is calculated as follows:

$$
d_{i, j}=\frac{1}{1+\left|z_{i}-z_{j}\right|}
$$

Since each $z_{i}$, for $i=1,2, \ldots, 4$, relates to other three components of $W_{(p, q)}$, shown in Fig.4, the associated weighting coefficient $w_{i}$ is given by

$$
w_{i}=\sum_{j=1, j \neq i}^{4} d_{i, j}
$$

Thus, the weighting coefficients $w_{i}$, for $i=1,2, \ldots, 4$, reflect the accuracy of the input components $z_{i}$ in terms of structural content of the Bayer data. When $d_{i, j}$ used in defining the weighting coefficients $w_{i}$ is small, no edge is positioned across the considered direction and the component $z_{i}$ is not penalized. If $z_{i}$ and $z_{j}$ are located across an edge, the corresponding absolute difference increases $d_{i, j}$ and the corresponding $z_{i}$ is penalized via $w_{i}$ appropriately. Thus, the weighting coefficients can be used to regulate the influence of $z_{1}, z_{2}, \ldots, z_{4}$, on the estimated value $z_{(p, q)}$ as follows:

$$
z_{(p, q)}=\frac{\sum_{i=1}^{4} w_{i} z_{i}}{\sum_{i=1}^{4} w_{i}}=\sum_{i=1}^{4} w_{i}^{\prime} z_{i}
$$

where $z_{1}, z_{2}, \ldots, z_{4}$ are corners of the used shape masks and $w_{i}^{\prime}=w_{i} / \sum_{j=1}^{4} w_{j}$, for $i=1,2, \ldots, 4$, are the normalized weighting coefficients. 


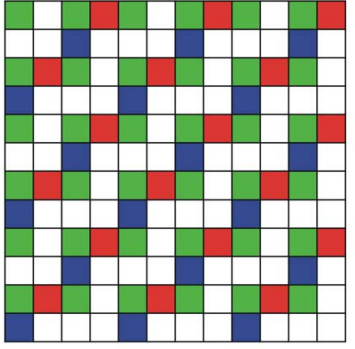

(a)

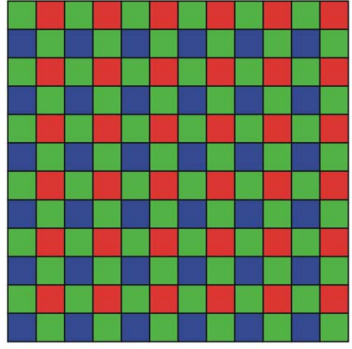

(b)
Fig. 5. Bayer images achieved using: (a) the first interpolation step, (b) the second interpolation step.

Based on a small corresponding weighting coefficient $w_{i}$, the missing $\mathrm{G}$ components $z_{(p, q)}$, or generally said missing components of the enlarged Bayer image $z$ are constituted with a small portion of $z_{i}$.

In (4), the normalized weight $w_{i}^{\prime}$ provides the degree to which a component $z_{i}$ contributes to the output. Through the normalization procedure, two constraints necessary to ensure that the output $z_{(p, q)}$ is an unbiased solution are satisfied. Namely,

1. Each weight is a positive number, $w_{i}^{\prime} \geq 0$.

2. The summation of all the weights is equal to unity $\sum_{i=1}^{4} w_{i}^{\prime}=1$.

Performing the interpolation step of (4), the enlarged Bayer image results in the pattern depicted in Fig.5a. This however, does not produce all the needed values and one more interpolation of the missing $\mathrm{G}$ components is necessary. This interpolation step produces the $\mathrm{G}$ components positioned in the center of the square-shape mask moved over the $z$ image domain and $\mathrm{R}$ (or $\mathrm{B}$ ) components in the center of the diamond-shape mask. Note that $z_{1}, z_{2}, z_{3}, z_{4}$ are equivalent to the $\mathrm{G}$ components $z_{(p-1, q-1)}, z_{(p-1, q+1)}$, $z_{(p+1, q-1)}$ and $z_{(p+1, q+1)}$, whereas the $\mathrm{R}$ (or B) components are interpolating using $z_{(p-2, q)}, z_{(p, q-2)}, z_{(p, q+2)}$ and $z_{(p+2, q)}$. This interpolation step completes the enlarged Bayer image $z$ shown in Fig.5b.

However, based on the CFA interpolation methodology, the $\mathrm{R}$ and $\mathrm{B}$ components can be interpolated more accurately using a difference color model of [1], which is frequently utilized in the CFA interpolation schemes [7]. Completing the $\mathrm{G}$ components, it can be observed that the missing $\mathrm{R}$ components $z_{(p, q)}$ occupy positions directly neighboring with the $\mathrm{G}$ components $z_{(p, q-1)}$ and the missing $\mathrm{B}$ components are neighbors of $z_{(p-1, q)}$. Similarly, the original $\mathrm{R}$ components $z_{1}, z_{2}, z_{3}, z_{4}$ corresponding to the samples $z_{(p-2, q-2)}, z_{(p-2, q+2)}, z_{(p+2, q-2)}$ and $z_{(p+2, q+2)}$ are directly neighboring with the $\mathrm{G}$ components $z_{1}^{\prime}, z_{2}^{\prime}, z_{3}^{\prime}, z_{4}^{\prime}$ given by $z_{(p-2, q-3)}, z_{(p-2, q+1)}, z_{(p+2, q-3)}, z_{(p+2, q+1)}$. In the case of the B components, the quantities $z_{1}^{\prime}, z_{2}^{\prime}, z_{3}^{\prime}, z_{4}^{\prime}$

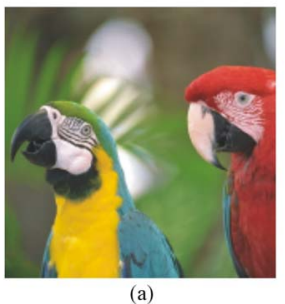

(a)

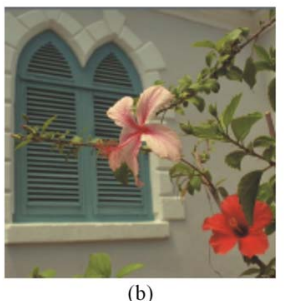

(b)
Fig. 6. Test color images: (a) Parrots, (b) Window.

denote the $\mathrm{G}$ components $z_{(p-3, q-2)}, z_{(p-3, q+2)}, z_{(p+1, q-2)}$, and $z_{(p+1, q+2)}$.

Assuming that the RGB color components are highly correlated, the normalized weighted sum of (4) is calculated using the differences between R and G (or B and G) components. The result of this operation has to be added to $\mathrm{G}$ components $z_{0}^{\prime}$ normalizing the interpolation output to the useful range:

$$
z_{(p, q)}=z_{0}^{\prime}+\frac{\sum_{i=1}^{6} w_{i}\left(z_{i}-z_{i}^{\prime}\right)}{\sum_{i=1}^{6} w_{i}}
$$

where $z_{0}^{\prime}=z_{(p, q-1)}$ and $z_{0}^{\prime}=z_{(p-1, q)}$ are used interpolating the $\mathrm{R}$ and $\mathrm{B}$ components, respectively.

Since this step produces the $\mathrm{R}$ and $\mathrm{B}$ components depicted in Fig.5a, the missing R and B components positioned in the centers of diamond-shape mask are again interpolated using the color-difference based interpolation procedure of (5). Note that $z_{1}, z_{2}, z_{3}, z_{4}$ correspond to $z_{(p-2, q)}$, $z_{(p, q-2)}, z_{(p, q+2)}$, and $z_{(p+2, q)}$. Interpolating the R components, the $\mathrm{G}$ components $z_{1}^{\prime}, z_{2}^{\prime}, z_{3}^{\prime}, z_{4}^{\prime}$ relate to $z_{(p-2, q-1)}$, $z_{(p, q-3)}, z_{(p, q+1)}$ and $z_{(p+2, q-1)}$, whereas interpolating the B components, the $\mathrm{G}$ quantities $z_{1}^{\prime}, z_{2}^{\prime}, z_{3}^{\prime}, z_{4}^{\prime}$ correspond to $z_{(p-3, q)}, z_{(p-1, q-2)}, z_{(p-1, q+2)}$, and $z_{(p+1, q)}$. This interpolation step results in the enlarged Bayer image $z$ shown in Fig.5b.

\section{EXPERIMENTAL RESULTS}

The proposed zooming method is tested using the color images shown in Fig.6. These images have been captured using highly professional three-sensor cameras or color scanners. In order to facilitate comparisons, the test images have been normalized to the standard $512 \times 512,8$-bit per channel RGB representation.

To measure the efficiency of the zooming methods objectively, we used the following approach. The process starts with the $K_{1} \times K_{2}$ original color image $\mathbf{o}$, down-sampled to the $K_{1} / 2 \times K_{2} / 2$ color image $\mathbf{o}^{\prime}$. This image is transformed into the $K_{1} / 2 \times K_{2} / 2$ Bayer image $z^{\prime}$. The $K_{1} \times K_{2}$ zoomed Bayer image $z$ is constituted using the CFA zooming technique. Applying the CFA interpolation scheme, the 
Table 1. Comparison of the presented zooming algorithms using the BI CFA interpolation scheme.

\begin{tabular}{ccccccc}
\hline Image & \multicolumn{3}{c}{ Parrots } & \multicolumn{3}{c}{ Window } \\
\hline Method & $M A E$ & $M S E$ & $N C D$ & $M A E$ & $M S E$ & $N C D$ \\
\hline LAZ & 5.82 & 158.9 & 0.0481 & 8.29 & 204.1 & 0.0908 \\
CCZ & 4.91 & 124.6 & 0.0388 & 7.02 & 149.9 & 0.0739 \\
Proposed & 4.98 & 113.0 & 0.0396 & 6.50 & 127.9 & 0.0747 \\
\hline
\end{tabular}

$K_{1} \times K_{2}$ output color image $\mathbf{y}$ is achieved. The similarity between $\mathbf{o}$ and $\mathbf{y}$ is measured via the mean absolute error (MAE), the mean square error (MSE) and the normalized difference criterion (NCD) [8].

The proposed method is compared to the locally adaptive CFA zooming (LAZ) of [3]. The output of these CFA zooming methods has been restored using well-known bilinear CFA interpolation. The proposed method is compared, in terms of efficiency with the conventional color image zooming (CCZ) approach (the BI CFA interpolation followed by the bicubic RGB zooming scheme).

Excellent design characteristics of the proposed CFA zooming scheme result in the best values of objective criteria (Table 1) among the tested zooming methods. On this place it has to be emphasized that the CFA zooming schemes employ a three-times less amount of data to be processed compared to the CCZ. Fig.7 presents zoomed parts of the restored images. These results allow the subjective evaluation of the restored images. Inaccurate zooming performance significantly affects the restored image quality and leads to strong color artifacts and blurred image edges.

\section{CONCLUSIONS}

A new zooming method operating on Bayer CFA data was introduced. The method employed edge sensing mechanism and difference color model. Combining their advantages, the proposed method enlarges Bayer images, while preserving edges and structural contents. Applying the CFA interpolation method, the introduced zooming algorithm produces enlarged color images pleasurable for viewing. At the same time it yields excellent results in terms of commonly used objective image quality criteria.

\section{REFERENCES}

[1] J. Adams, "Design of practical color filter array interpolation algorithms for digital cameras," Proc. SPIE, vol. 3028, pp. 117-125, February 1997.

[2] B.E. Bayer, "Color imaging array," U.S. Patent 3971 065, 1976.

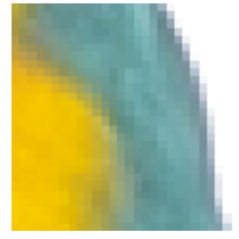

(a)

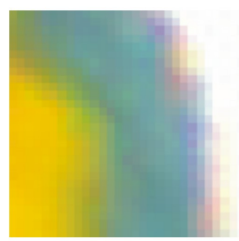

(c)

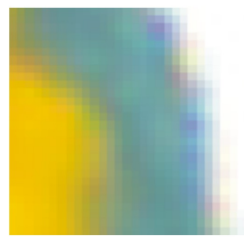

(e)

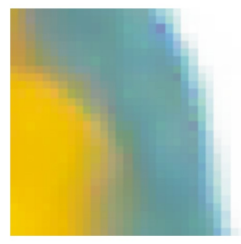

(g)

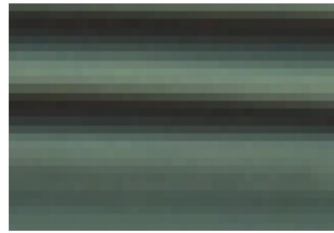

(b)

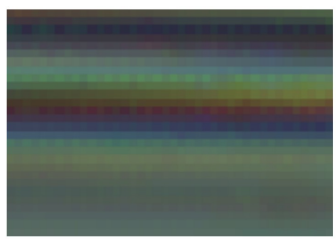

(d)

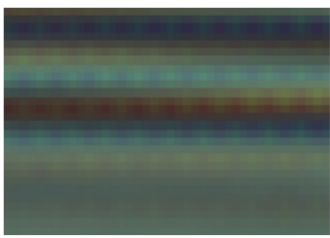

(f)

(h)

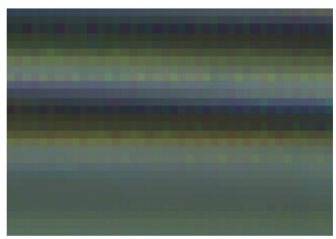

Fig. 7. Zoomed results corresponding to Fig.6: (a,b) original image, (c,d) LAZ approach, (e,f) CCZ approach, (g,h) proposed zooming approach.

[3] S. Battiato, G. Gallo, and F. Stanco, "A locally adaptive zooming algorithm for digital images," Image and $\mathrm{Vi}$ sion Computing, vol. 20, pp. 805-812, September 2002.

[4] A. Bosco, M. Mancuso, S. Battiato, and G. Spampinato, "Adaptive temporal filtering for CFA video sequences," Proc. ACIVS'02, pp. S00:1-6, September 2002.

[5] C.C. Koh and S.K. Mitra, "Compression of Bayer color filter array data," Proc. ICIP'03, vol. 2, pp. 255-258, September 2003.

[6] R. Lukac and K.N. Plataniotis, "Digital camera zooming on the colour filter array," IEE Electronics Letters, vol. 39, pp. 1806-1807, December 2003.

[7] S.C. Pei and I.K. Tam, "Effective color interpolation in CCD color filter arrays using signal correlation," IEEE Circ. Sys. Video Tech., vol. 13, pp. 503-513, June 2003.

[8] K.N. Plataniotis and A.N. Venetsanopulos, Color Image Processing and Applications, Springer-Verlag, 2000. 